\title{
A Level Set Filter for Speckle Reduction in SAR Images
}

\author{
Hongga Li, ${ }^{1}$ Bo Huang, ${ }^{2}$ and Xiaoxia Huang1 \\ ${ }^{1}$ Institute of Remote Sensing Applications, Chinese Academy of Sciences, Beijing 100101, China \\ ${ }^{2}$ Department of Geography and Resource Management, The Chinese University of Hong Kong, Shatin, NT, Hong Kong
}

Correspondence should be addressed to Bo Huang, bohuang@cuhk.edu.hk

Received 11 June 2009; Revised 15 December 2009; Accepted 12 February 2010

Academic Editor: Carlos Lopez-Martinez

Copyright ( 92010 Hongga Li et al. This is an open access article distributed under the Creative Commons Attribution License, which permits unrestricted use, distribution, and reproduction in any medium, provided the original work is properly cited.

\begin{abstract}
Despite much effort and significant progress in recent years, speckle removal for Synthetic Aperture Radar (SAR) image still is a challenging problem in image processing. Unlike the traditional noise filters, which are mainly based on local neighborhood statistical average or frequencies transform, in this paper, we propose a speckle reduction method based on the theory of level set, one form of curvature flow propagation. Firstly, based on partial differential equation, the Lee filter can be cast as a formulation of anisotropic diffusion function; furthermore, we continued to deduce it into a level set formulation. Level set flow into the method allows the front interface to propagate naturally with topological changes, where the speed is proportional to the curvature of the intensity contours in an image. Hence, small speckle will disappear quickly, while large scale interfaces will be slow to evolve. Secondly, for preserving finer detailed structures in images when smoothing the speckle, the evolution is switched between minimum or maximum curvature speed depending on the scale of speckle. The proposed method has been illustrated by experiments on simulation image and ERS-2 SAR images under different circumstances. Its advantages over the traditional speckle reduction filter approaches have also been demonstrated.
\end{abstract}

\section{Introduction}

Due to some unique characteristics of Synthetic Aperture Radar (SAR), such as all-weather, capability of penetrating cloud cover, and special reflect spectrum for object, this gives it a considerable advantage over other infrared or optical sensors. Meanwhile, it accelerates SAR applications for environmental monitoring. However, the imagery produced by SAR systems is degraded by a high level of noise due to the inherent nature of radar backscatter. The largest source of noise in a SAR image is the speckle noise that is caused by the interaction of the coherent imaging radar beam and the relatively rough surface being imaged. Because of the speckle, a form of multiplicative, locally correlated noise, typical image processing techniques experience a great difficulty when applied to SAR imagery.

In order to tackle this problem for SAR image processing, many methods have been attempted. The most widely cited and applied in the SAR community are based on the minimum mean square error (MMSE) theory, and mainly focused on the geometric domain, including Lee [1], Enhanced Lee [2], Kuan [3], Frost [4], Enhanced Frost
[2] filters. At the same time, more and more diffusion and random filters are put forwards to reduce SAR image speckle, such as anisotropic diffusion [5], nonlinear diffusion [6], speckle reducing anisotropic diffusion (SRAD) [7], detail preserving anisotropic diffusion (DPAD) [8], wavelet processing [9], Markov Random Fields [10], and hysteresis thresholding [11].

Most of above traditional speckle filters, which mainly rely on local adaptive statistical data related to the filtered pixel, have been proposed to reduce speckle, the main difficulty being to suppress speckle while preserving image information such as edges or textures [12]. On one hand, because the results depend on the occurrence of the filter window over an area, the achievement of both speckle reduction and preservation of edge data is only possible when the filter window happens to cover an area that is uniform. On the other hand, traditional filters are not directional. Thus, more and more edge preserving techniques were developed and applied in to optimize MMSE speckle filtering [13]. For example, ratio edge detector [2] and the evolution of the coefficient of variation [12] have been validated with the MMSE for detecting edge and maximum 
homogeneous area for speckle smoothing, respectively. For polarization SAR images, the classification map of scattering characteristics is used with speckle filtering of polarimetric synthetic aperture radar (POLSAR) filter [14] for speckle reduction, while perfectly preserving strong point target signatures, and retains edges, linear, and curved features. Meanwhile, for multitemporal SAR image filtering, a constant false-alarm rate (CFAR) edge detector and conventional statistical criteria are integrated for spatial and temporal information preservation [15]. In addition, for the diffusion speckle removal approach reduces noise and texture but also preserves and enhances edges, the diffusion formulations such as anisotropic diffusion [5], nonlinear diffusion [6], SRAD [7], DPAD [8] are becoming an important way for edge-sensitive speckle smoothing.

In order to not only remove speckle but also keep edge, an alternative approach is proposed in this paper, it mainly relies on the theory of curvature anisotropic flow, that is, level set method. Firstly, the Lee filter can be transformed as a formulation of anisotropic diffusion function [7]. Thus, after the analysis, the relationship between curvature flow and Lee speckle filter, we considered further and deduced the anisotropic diffusion filter into a formulation of level set. Therefore, the new technique is not only based on the minimum mean square error approach to filtering as the Lee filters, but also on anisotropic diffusion sensitive to edges. The paradigm of the level set is a numerical method for tracking the evolution of contours and surfaces. In this approach, an image is interpreted as a collection of iso-intensity contours. Similar to anisotropic diffusion, under a curvature-driven factor, smoothing takes place inside a region, but not across region boundaries. So the attractive quality of this approach is that sharp boundaries are preserved while speckles are smoothed. Secondly, in order to avoid long-term iteration in the manner of traditional anisotropic diffusion, the different speed of flow approach was introduced into the new technique as a selective smoothing switch according to effects by intensity and curvature gradient of neighbor pixels. An image is filtered as a set of iso-intensity contours which can be evolved. The spikes of noise correspond to high curvature objects, so these are easily reduced by flow propagation under curvature; meanwhile, oscillations in boundaries are smoothed out, all the while preserving sharp boundaries.

The rest of this paper is organized as follows. In Section 2, we first review the background of level set and the main SAR image noise removal algorithms. In Section 3, we propose new filter algorithm based on level set and Lee filter, including processing flowchart, filter characteristics, and the flow speeds switch control. Section 4 presents the results of experimental evaluation of simulation image and SAR images, respectively. Finally, Section 5 summarizes this proposed method.

\section{Background}

During the last 20 years, many speckle filters for SAR images were developed and documented. Speckle noise is a common phenomenon in all coherent imaging systems like laser, acoustic, and SAR imagery. The source of this noise is attributed to random interference between the coherent returns issued from the numerous scatterers present on a surface, on the scale of a wavelength of the incident radar. Speckle noise gives the SAR image a grainy appearance and prevents target recognition and texture analysis efficiently. Obviously, speckle filtering turns out to be an important preprocessing step for some detection or classification optimization. Because speckle noise has the characteristics of a random multiplicative, thus traditional filtering will not remove it easily. In practice, the most widely used filters such as Lee (Enhanced Lee), Kuan and Frost filters are based on a Minimum Mean Square Error (MMSE) criterion. Thus, in this section, we briefly describe the representative MMSE method, that is, Lee filter and background of level set.

2.1. Lee Filter. The Lee filter, derived from both additive and multiplicative noise, is a very popular statistical filter, which is designed to eliminate speckle noise while preserving edges and point features in SAR imagery. Based on Minimum Mean Square Error (MMSE) criterion, a smoothed pixel for multiplicative noise model can be represented as follows

$$
\hat{I}_{(x, y)}=\bar{I}_{s}+k_{s}\left(I_{(x, y)}-\bar{I}_{s}\right)
$$

where $\bar{I}_{s}$ is the mean value of the intensity within the filter window $\eta_{s}$, and $k_{s}$ is the adaptive filter coefficientdetermined by

$$
k_{s}=1-\frac{C_{u}^{2}}{C_{s}^{2}}
$$

Here,

$$
C_{s}^{2}=\frac{\left(1 /\left|\eta_{s}\right|\right) \sum_{x, y \in \eta}\left(I_{(x, y)}-\bar{I}_{s}\right)^{2}}{\left(I_{(x, y)}-\bar{I}_{s}\right)^{2}}
$$

and $C_{u}^{2}$ is a constant for a given image and can be determined by either

$$
C_{u}^{2}=\frac{\operatorname{var}\left(z^{\prime}\right)}{\left(\bar{z}^{\prime}\right)^{2}}, \quad \text { or } C_{u}^{2}=\frac{1}{L}
$$

where $L$ is the effective number of looks of the noisy image, and $\operatorname{var}\left(z^{\prime}\right)$ and $\bar{z}^{\prime}$ are the intensity variance and mean over a homogeneous area of image, respectively. Obviously, in uniform areas, the value ofC $\mathrm{s}_{s}$ approachesC $\mathrm{C}_{u}$ and $k_{s}$ nears to zero, leading to the same result as mean filter. At edges, the value ofC $\mathrm{s}_{s}$ becomes larger, and $k_{s}$ nears to unity, resulting in little modification.

Kuan filter is considered to be more efficient than Lee filter. The resulting filter for signal dependent noise is the same as Lee filter except for the $k_{s}$, which is defined as

$$
k_{s}=\frac{\bar{z}^{\prime} \operatorname{var}\left(I_{s}\right)}{\bar{I}_{s}^{2} \operatorname{var}\left(z^{\prime}\right)+\bar{z}^{\prime 2} \operatorname{var}\left(I_{s}\right)+\operatorname{var}\left(z^{\prime}\right) \operatorname{var}\left(I_{s}\right)} .
$$


2.2. Level Set Method. Level set, introduced by Osher and Sethian [16], is an efficient numerical technique for interface propagation, which we have introduced in SAR images segmentation $[17,18]$. A brief introduction of this method is given here. The detailed explanation can be found in Sethian [19].

In the level set method, a scalar Lipschitz function, $\Psi(x, t)$ (also known as level set function), defines the embedding of an $n$-dimensional surface in an $R^{n+1}$ space surface, where $x \in R^{n+1}$, and $t=$ time $t$. The set of points on the surface, $\Gamma$, are mapped by $\Psi$ such that

$$
\Gamma((x), t)=\{\Psi(x, t)=k\},
$$

where $k$ is an arbitrary scalar value, namely, $\Gamma$ is the $k$ level set of $\Psi$.

The essential idea of the level set is to represent the moving front $\partial \Psi$ as the zero level set of the time-dependent level set function $\Psi$. In accordance with the propagation of the front, the partial differential equation (PDE) of general level set is represented as

$$
\begin{aligned}
\frac{\mathrm{d} \Psi}{\mathrm{d} t} & =F|\nabla \Psi| \\
& =-\alpha A(x) \cdot \nabla \Psi-\beta P(x)|\nabla \Psi|+\gamma Z(x) \kappa|\nabla \Psi|,
\end{aligned}
$$

where $F$ is a scalar function that defines the speed in the outward direction normal to $\Psi,|\nabla \Psi|$ represents some appropriate finite different operators for the spatial derivative, $\mathrm{d} t$ is the time step, the symbol • stands for the scalar product, and I| denotes the magnitude. Traditionally, the speed in which the front propagates is defined by the function $F$, which may be dependent on many factors, $F=F(\mathrm{~A}, \mathrm{P}, \mathrm{Z}) . \mathrm{A}$ is an advection term, $\mathrm{P}$ is a propagation (expansion) term, and $Z$ is a spatial modifier term for the mean curvature $\kappa$; the scalar constants, $\alpha$, $\beta$, and $\gamma$ weight the relative influence of each of the terms on the movement of the interface. In general, the level set method provides a technique to deform the implicit surface continuously following the gradient descent of the energy functional to reach the object boundary. The mean curvature $\kappa$ is defined as

$$
\kappa=\nabla \cdot \frac{\nabla \Psi}{|\nabla \Psi|}
$$

When level set is used into image process such as segment or noise removal, the level set function $\Psi$ is replaced by pixel intensity $I$. In other words, an image is interpreted as a collection of iso-intensity contours which are viewed as level sets, where pixels of a particular intensity form one level set. The relation between level set curvature function and partial differential in the image process is represented as

$$
I_{t}=\nabla^{2} I=F|\nabla I| \text {. }
$$

The key of level set for image processing is to define reasonable scalar function $F$. For SAR image, intensity model and curvature model are integrated to improve effectiveness. And in the previous image filter, scalar function $F$ is usually simplified to $F=\kappa /|\nabla I|[20]$ or $F=\kappa^{1 / 3}[21]$.

\section{The Proposed Level Set Filter}

Referring to the above Lee filter theory and level set numerical approach, in this section we derive a new level set method from traditional Lee filter for spackle noise reduction in SAR image.

3.1. The Level Set Formulation for Speckle Reduction. As we know, the Lee filter, or Kuan filter processes a current pixel based on its intensity and neighboring pixels inside a fixed square window. Based on Lee filter,Yu and Actom [7] developed an anisotropic diffusion scheme.

3.1.1. From Lee Filter to Discrete Isotropic Diffusion Equation. Typically, the Lee filter operates in a $7 \times 7$ moving window. Imaging choosing the filter windows as $\eta_{s}=\overline{\eta_{s}} \equiv\{(i-$ $1, j),(i, j-1),(i+1, j),(i, j+1)\}$ at an interior site $s=(i, j)$, the special case of the Lee filter with window is expressed in the following diffusion form:

$$
\begin{aligned}
\widehat{I}_{i, j} & =\bar{I}_{\eta_{s}}+k_{i, j}\left(I_{i, j}-\bar{I}_{\eta_{s}}\right) \\
& =I_{i, j}+\left(1-k_{i, j}\right)\left(\bar{I}_{\eta_{s}}-I_{i, j}\right) \\
& =I_{i, j}+\left(1-k_{i, j}\right)\left(\frac{1}{\left|\bar{\eta}_{s}\right|} \sum_{p \in \eta_{s}} I_{p}-I_{i, j}\right) \\
& =I_{i, j}+\left(1-k_{i, j}\right)\left(\frac{1}{\left|\bar{\eta}_{s}\right|} \sum_{p \in \eta_{s}}\left(I_{p}-I_{i, j}\right)\right) \\
& =I_{i, j}+\left(1-k_{i, j}\right) \frac{1}{\left|\bar{\eta}_{s}\right|} \nabla^{2} I_{s, p},
\end{aligned}
$$

where $\overline{\eta_{s}}$ represents the spatial neighborhood of pixel $s, \overline{\left|\eta_{s}\right|}$ is the number of pixels in the window (usually four), and $\nabla I_{s, p}=I_{p}-I_{s}, \forall p \in \overline{\eta_{s}}$. Consider the differential iteration, if we substitute $I_{i, j}^{n+1}, I_{i, j}^{n}, k_{i, j}^{n}$ for $\hat{I}_{i, j}, I_{i, j}, k_{i, j}$ in (10), respectively, we obtain

$$
I_{i, j}^{n+1}=I_{i, j}^{n}+\left(1-k_{i, j}^{n}\right) \frac{1}{\left|\bar{\eta}_{s}\right|} \nabla^{2} I_{i, j}^{n}
$$

Thus, traditional Lee filter firstly is represented as a discrete isotropic diffusion equation.

3.1.2. From Lee Filter to Discrete Anisotropic Diffusion Equation. Moreover, according toYu and Acton [7] scheme, these above functions show the effect of assigning slightly different weights to the four directional differences in (12) 


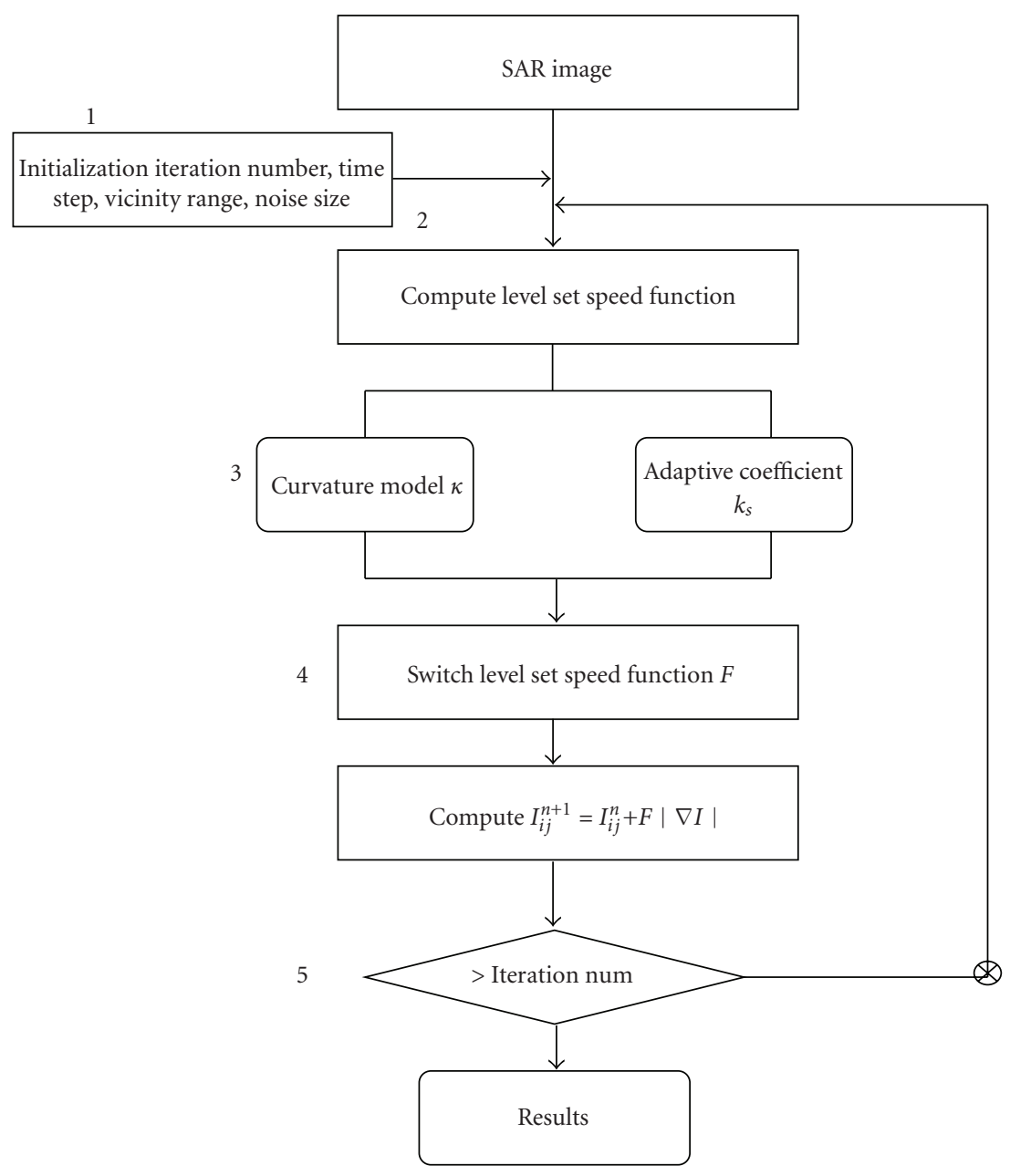

FIGURE 1: The framework for proposed level set filter

as follows:

$$
\begin{aligned}
\hat{I}_{i, j}=I_{i, j}+\left(1-k_{i, j}\right) \frac{1}{\left|\bar{\eta}_{s}\right|} \nabla^{2} I_{i, j} \\
=I_{i, j}+\frac{1}{\left|\bar{\eta}_{s}\right|}\left[\left(1-k_{i+1, j}\right)\left(I_{i+1, j}-I_{i, j}\right)\right. \\
+\left(1-k_{i, j}\right)\left(I_{i-1, j}-I_{i, j}\right) \\
+\left(1-k_{i, j}+1\right)\left(I_{i, j+1}-I_{i, j}\right) \\
\left.+\left(1-k_{i, j}\right)\left(I_{i, j-1}-I_{i, j}\right)\right] .
\end{aligned}
$$

In fact, (12) reduces to

$$
\widehat{I}_{i, j}=I_{i, j}+\frac{1}{\left|\bar{\eta}_{s}\right|} \operatorname{div}\left[\left(1-k_{i, j}\right) \nabla I_{i, j}\right],
$$

where div is the divergence operator, and $\nabla$ the gradient operator. The above modification of the Lee filter is a form of anisotropic diffusion with $n=1$. The Lee filter processes a current pixel based on its intensity and intensities of neighboring pixels inside a fixed square window without mechanism to enhance edges or feature structures within a window.

By extending the PDE versions of the despeckle filters, a more general update function is represented as

$$
I_{i, j}^{t+\Delta t}=I_{i, j}^{t}+\frac{\Delta t}{\left|\bar{\eta}_{s}\right|} \operatorname{div}\left[c\left(C_{i, j}^{t}\right) \nabla^{2} I_{i, j}^{t}\right],
$$

where $c(x)$ is the diffusion coefficient, and $C_{i, j}$ is usually called local coefficient of variation. According to the above function, $\mathrm{Yu}$ and Acton [7] deduced local coefficient of variation as follows:

$$
C_{2 i, j}^{2}=\frac{(1 / 2)\left|\nabla I_{i, j}\right|^{2}-(1 / 16)\left(\nabla^{2} I_{i, j}\right)^{2}}{\left[I_{i, j}+(1 / 4) \nabla^{2} I_{i, j}\right]^{2}} .
$$




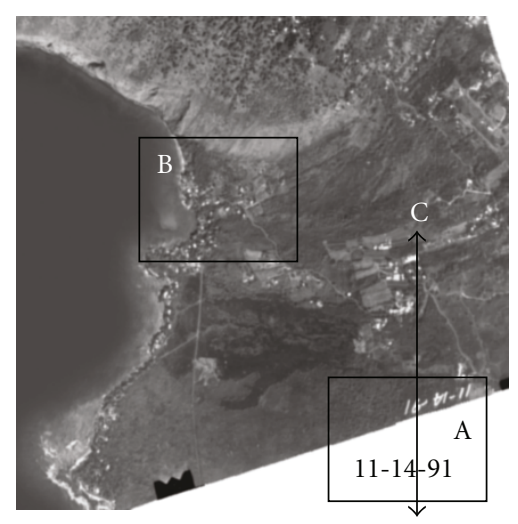

(a) Raw image

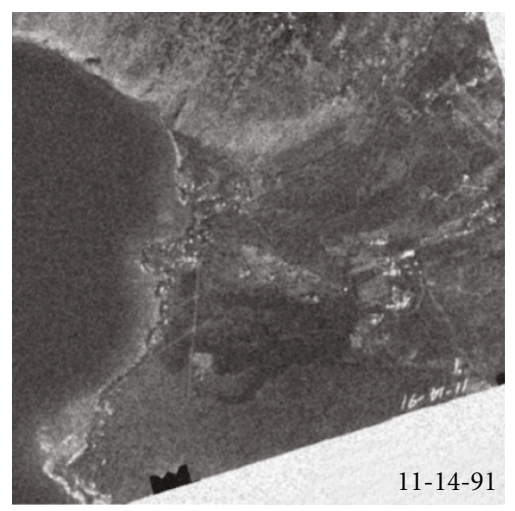

(d) Enhanced Lee Filter

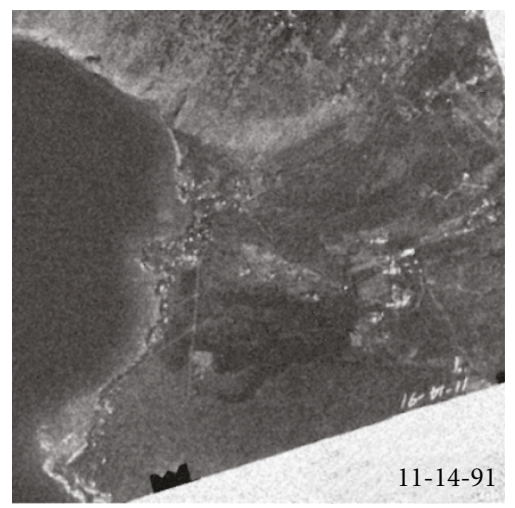

(g) Enhanced Frost Filter

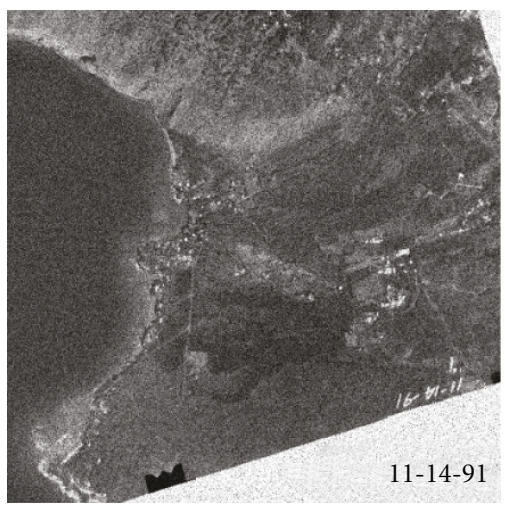

(b) Degraded image by speckle

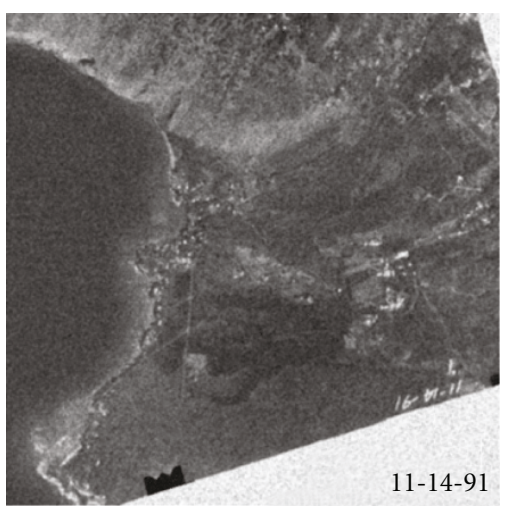

(e) Kuan Filter

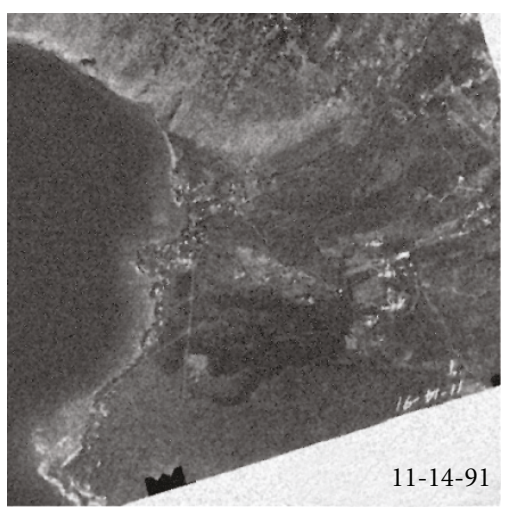

(h) Gamma Filter

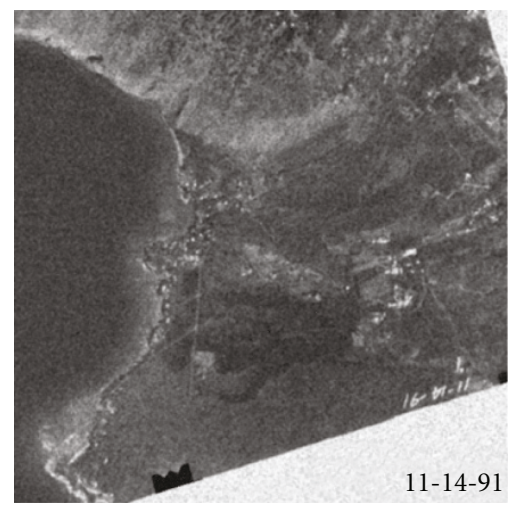

(c) Lee Filter

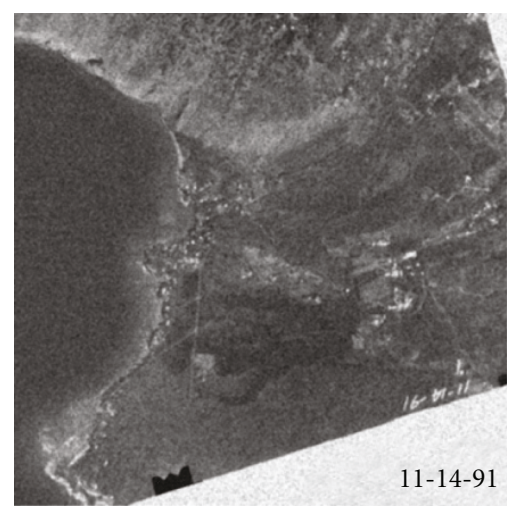

(f) Frost Filter

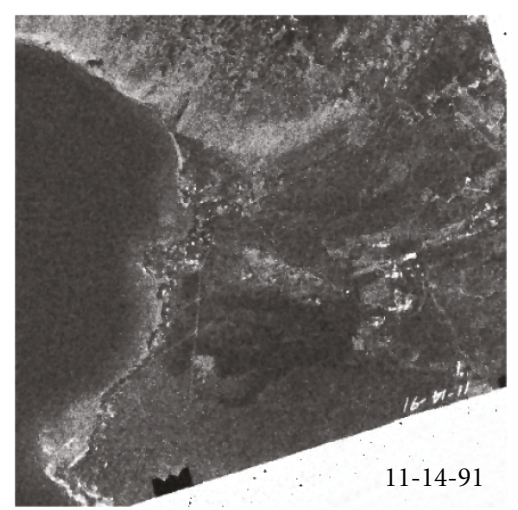

(i) Level set Filter

FIGURE 2: The proposed level set-based filter in contrast to the conventional approaches of simulation image.

The above anisotropic diffusion combines a normalized gradient magnitude operator and laplacian operator to act like edge detector for speckled imagery. The above equations from (10) to (15) are cited from the paper byYu and Acton [7] and the detailed deduction refers to their anisotropic diffusion scheme. In addition, AjaFernandez and Alberola-Lopez [8] modified the SRAD filter to rely on the Kuan filter rather than the Lee filter, and estimated the local statistics using a larger neighborhood than the 4 direct neighbors, showing that better results and better stability can be obtained using a $5 \times 5$ neighborhood.
3.1.3. From Lee Filter to Level Set Filter. In this paper, substituting the level set speed function (9) for diffusion part into Lee filter discrete isotropic diffusion (10), we obtain a base level set alternative approach of Lee filter:

$$
\begin{aligned}
\widehat{I}_{i, j} & =I_{i, j}+\left(1-k_{i, j}\right) \frac{1}{\left|\bar{\eta}_{s}\right|} \nabla^{2} I_{s, p} \\
& =I_{i, j}+\left(1-k_{i, j}\right) \frac{1}{\left|\bar{\eta}_{s}\right|} F\left|\nabla I_{(i, j)}\right| .
\end{aligned}
$$




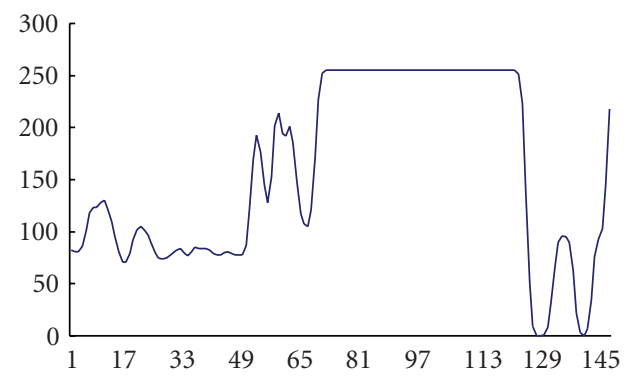

(a) Raw Data

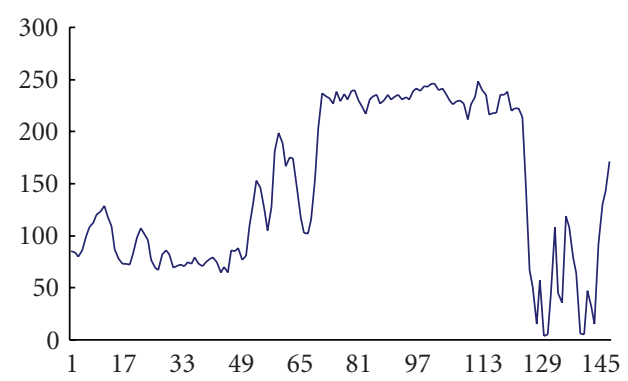

(c) Lee Filter

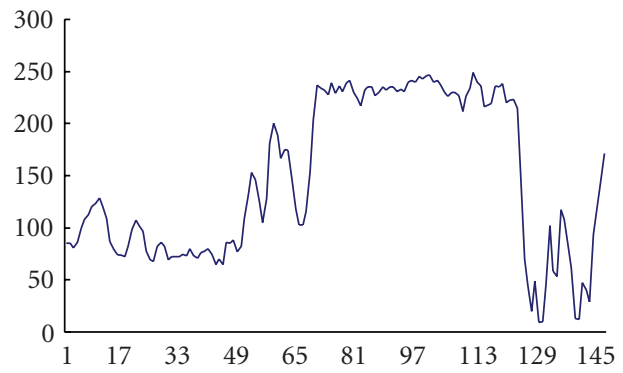

(e) Kuan Filter

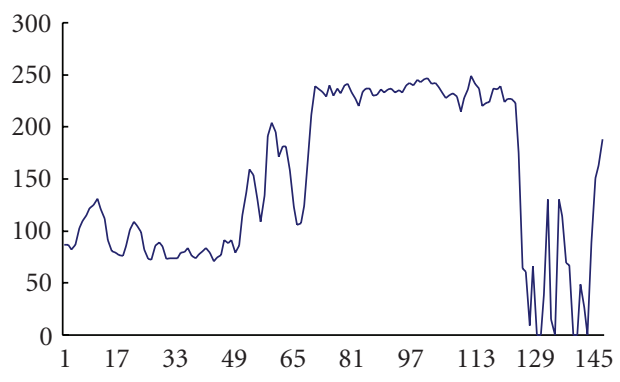

(g) Enhanced Frost Filter

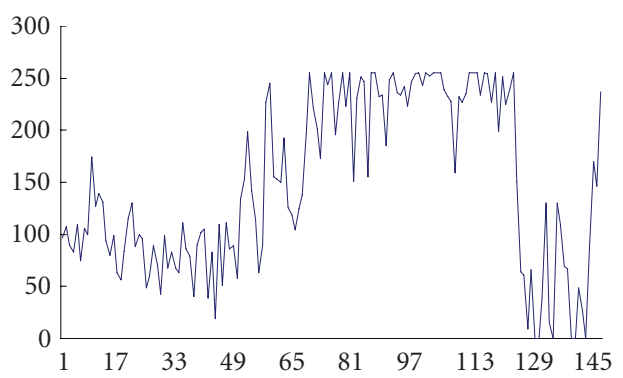

(b) Degraded image by speckle

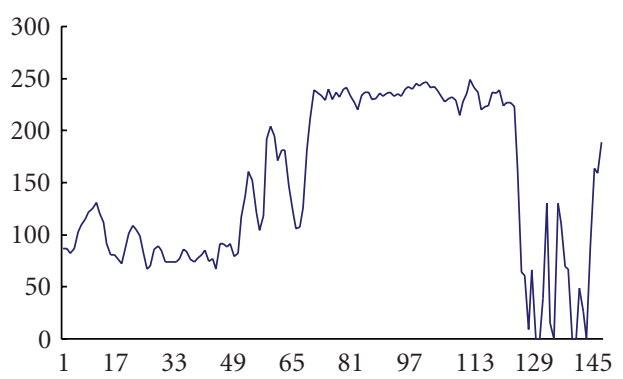

(d) Enhanced Lee Filter

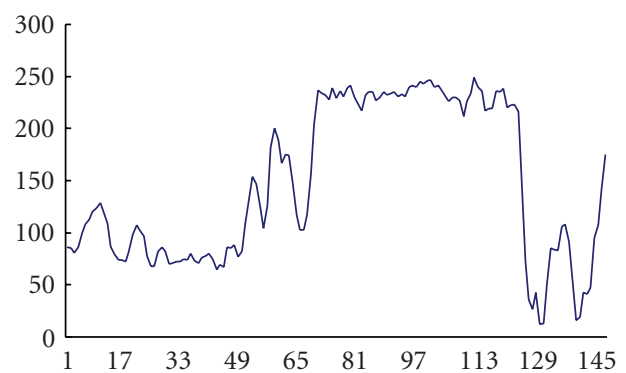

(f) Frost Filter

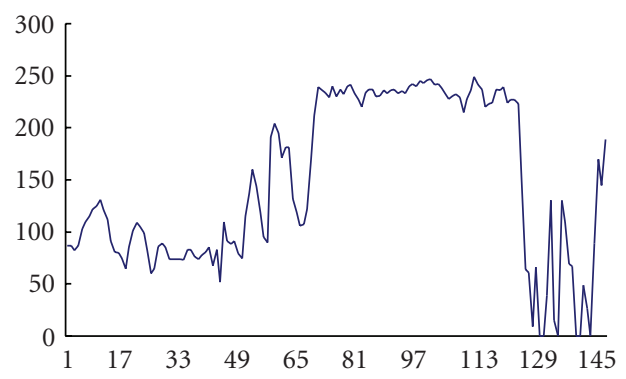

(h) Gamma Filter

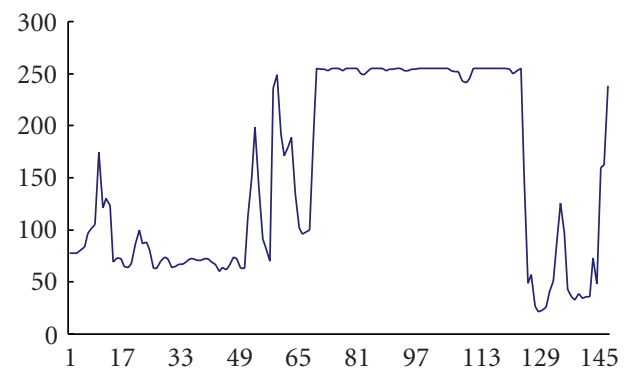

(i) Level set Filter

FIgure 3: The profiles of Region C resulted from the proposed level set-based filter and conventional approaches. 
Meanwhile, ignoring the advection and propagation term in the image process, the speed is represented as

$$
F=\nabla \cdot \frac{\nabla \Psi}{|\nabla \Psi|}=\kappa
$$

Considering the numerical solutions of level set function, if we substitute $I_{i, j}^{n+1}, I_{i, j}^{n}, k_{i, j}^{n}$ for $\widehat{I}_{i, j}, I_{i, j}, k_{i, j}$ in (16), respectively, by extending the propagation partial diffusion equation, we obtain the numerical approximation differential equation with the time step $\Delta t$ :

$$
\begin{aligned}
& I_{i, j}^{n+1}=I_{i, j}^{n}+\Delta t\left(1-k_{i, j}^{n}\right) \frac{1}{\left|\bar{\eta}_{s}\right|} F\left|\nabla I_{i, j}^{n}\right|, \\
& I_{i, j}^{n+1}=I_{i, j}^{n}+\Delta t\left(1-k_{i, j}^{n}\right) \frac{1}{\left|\bar{\eta}_{s}\right|} \kappa\left|\nabla I_{i, j}^{n}\right| .
\end{aligned}
$$

As a result, we obtained a curvature-flow-driven level set filter from MMSE Lee filter technique in the manner of partial diffusion equation, in other words we deduced the level set numerical form of traditional Lee filter. Obviously, the interface $|\nabla \Psi|$ represents appropriate finite difference operator for the spatial derivate, so calculation anisotropically takes place in different directions. The modification including directional sensitivity and filtering perpendicular to the edge direction would significantly improve reducing the speckle in the vicinity of edges and small features.

Areas of high curvature will diffuse faster than areas of low curvature. Hence, small jagged noise artifacts will disappear quickly, while large-scale interfaces will be slow to evolve. Moreover, the front of level set equals zero. Thus, the attractive quality of this approach is that sharp boundaries are preserved; smoothing takes place inside a region, but not across region boundaries, thereby preserving sharp boundaries between objects.

3.2. Curvature-Driven Model. Because the curvature plays important role in the proposed method, in this section, we detailed the numerical solution of curvature model by PDE. In order to obtain the stability of a boundary, an upwind partial differential scheme is employed to compute curvature value, which relies on a one-sided derivative that looks in the up-wind direction of the moving wave front, and thus avoids the over-shooting associated with finite forward differences.

Three difference operators, that is, the forward difference $D^{+}$, backward difference $D^{-}$, and central difference, are used in the eight neighboring pixels. For instance, the differences in the $x$ direction on the SAR image with spacing $h$ at time $t$, that is, $u(x, y, t)$, are defined by first- and second-order terms as

$$
\begin{gathered}
D_{x}^{+} u(x, y, t)=\frac{u(x+h, y, t)-u(x, y, t)}{h}, \\
D_{x}^{-} u(x, y, t)=\frac{u(x, y, t)-u(x-h, y, t)}{h}, \\
D_{x} u(x, y, t)=\frac{u(x+h, y, t)-u(x-h, y, t)}{2 h}, \\
D_{x}^{+y} u(x, y, t)=\frac{u(x+h, y+h, t)-u(x-h, y+h, t)}{2 h}, \\
D_{x}^{-y} u(x, y, t)=\frac{u(x+h, y-h, t)-u(x-h, y-h, t)}{2 h}, \\
D_{y}^{+x} u(x, y, t)=\frac{u(x+h, y+h, t)-u(x+h, y-h, t)}{2 h}, \\
D_{y}^{-x} u(x, y, t)=\frac{u(x-h, y+h, t)-u(x+h, y-h, t)}{2 h},
\end{gathered}
$$

where $D_{x}^{+}$computes the new value at $x, y$, and $x+h, y$; thus information for the solution propagates from right to left. $D_{x}^{-}$ computes the new value at $x, y$, and $x-h, y$; thus information for the solution propagates from left to right. $D_{x}$ computes the new value at $x+h, y$ and $x-h, y$, thus information for the solution propagates from both sides.

Meanwhile, the curvature is computed using the above derivatives and the difference of the normals method introduced by Whitaker and Xue [22]. The two normals, $n^{+}$and $n^{-}$, are computed by:

$$
\begin{aligned}
& n^{+}=\left\{\begin{array}{c}
\frac{D_{x}^{+}}{4 \sqrt{\left(D_{x}^{+}\right)^{2}+\left(\left(D_{y}^{+x}+D_{y}\right) / 2\right)^{2}}} \\
\frac{D_{y}^{+}}{4 \sqrt{\left(D_{y}^{+}\right)^{2}+\left(\left(D_{x}^{+y}+D_{x}\right) / 2\right)^{2}}}
\end{array}\right\}, \\
& n^{-}=\left\{\begin{array}{c}
\frac{D_{x}^{-}}{4 \sqrt{\left(D_{x}^{-}\right)^{2}+\left(\left(D_{y}^{-x}+D_{y}\right) / 2\right)^{2}}} \\
\frac{D_{y}^{-}}{4 \sqrt{\left(D_{y}^{-}\right)^{2}+\left(\left(D_{x}^{-y}+D_{x}\right) / 2\right)^{2}}}
\end{array}\right\} .
\end{aligned}
$$

The components of the divergence are then computed as

$$
\begin{aligned}
& \frac{\partial n_{x}}{\partial x}=n_{x}^{+}-n_{x}^{-}, \\
& \frac{\partial n_{y}}{\partial y}=n_{y}^{+}-n_{y}^{-} .
\end{aligned}
$$

Finally, the curvature model is formulated as

$$
\kappa=\frac{\partial n_{x}}{\partial x}+\frac{\partial n_{y}}{\partial y} .
$$




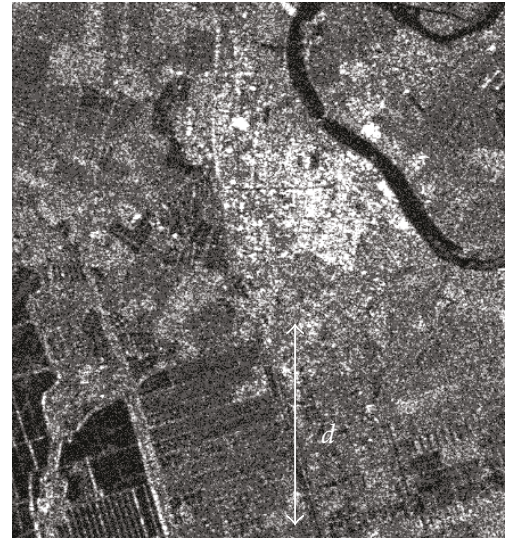

(a) Raw data

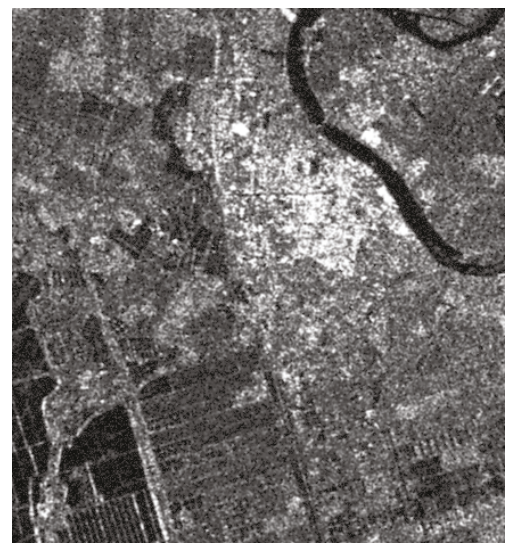

(d) Kuan Filter

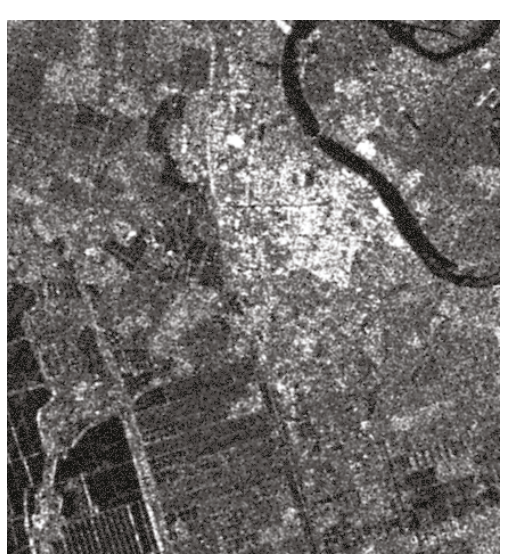

(b) Lee Filter

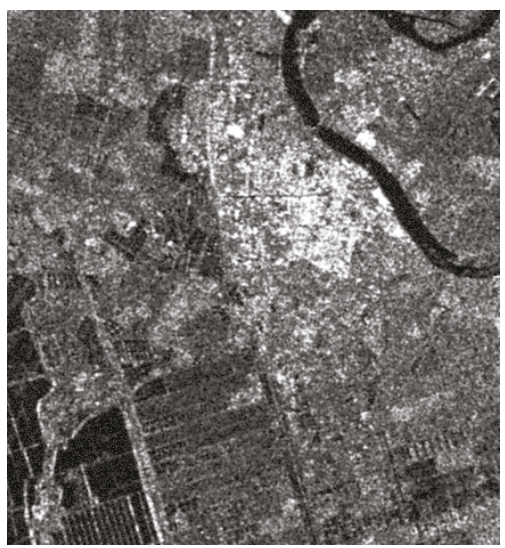

(e) Frost Filter

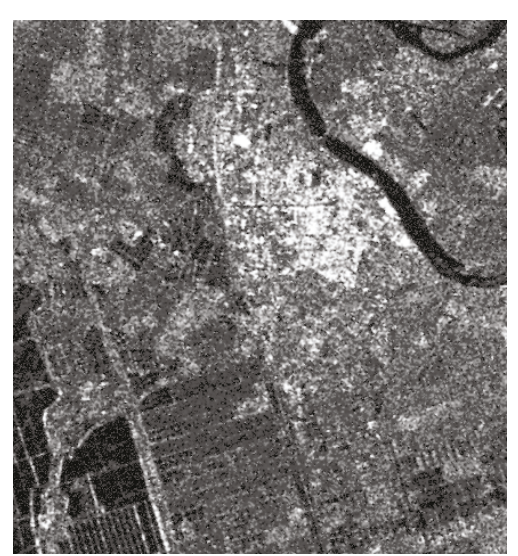

(c) Enhanced Lee Filter

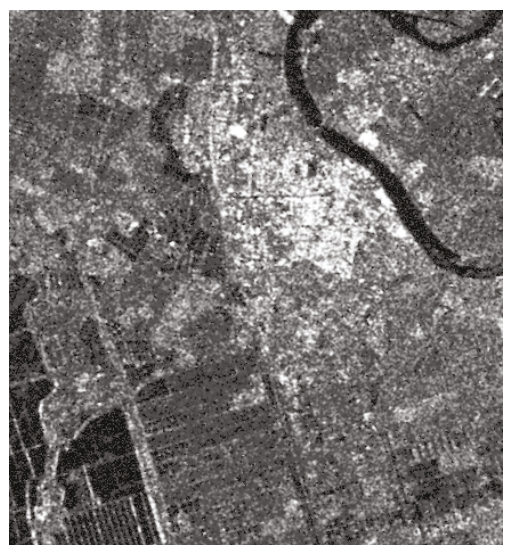

(f) Enhanced Frost Filter

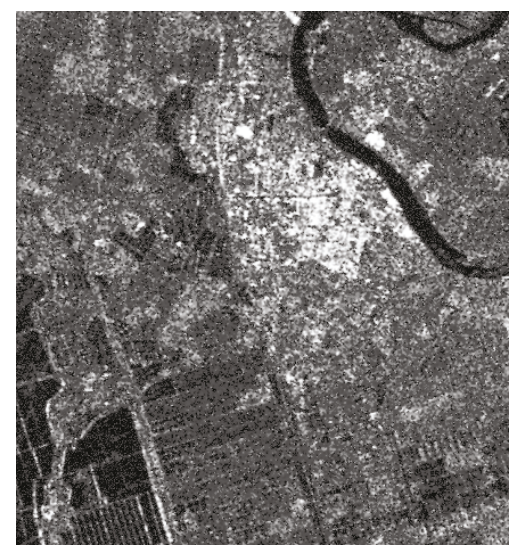

(g) Gamma Filter

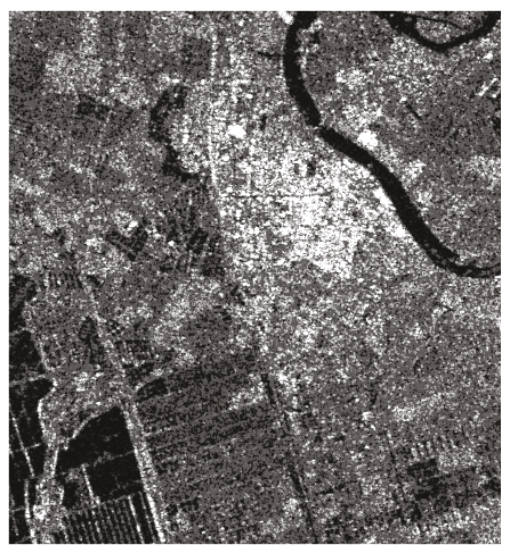

(h) Level set filter

FIGURE 4: The proposed level set-based filter in contrast to the conventional approaches of landscape ERS-2 SAR image.

With this equation, the direction and velocity of the speed dependent on the curvature can be easily derived.

Smoothing process anisotropically takes place in different directions. The speed function plays a central role in the level set as its value and normal direction determine the propagation velocity and direction of the front interface.

3.3. Min-Max Curvature Flow. According to function (16), unlike the common filters based on statistical average, the proposed approach relies on propagation diffusion iteration method. It should be noted that although the evolution at the boundary is slow, some diffusion still occurs. Thus, according to Grayson's theorem, continual application of this curvature flow scheme will eventually result in the removal of information as each contour shrinks to a point and disappears. So, we used the min-max flow [19] for preserving finer detailed structures in images from oversmoothing. 


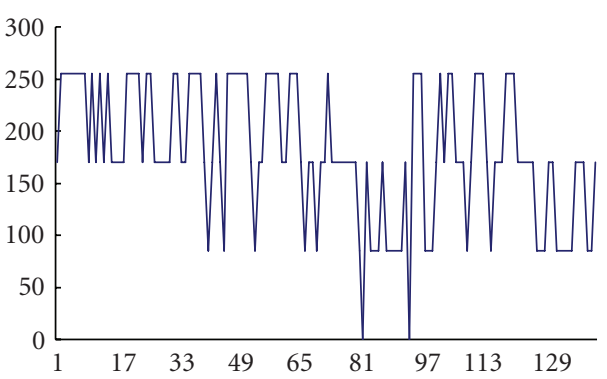

(a) Raw data, mean $=130.986, \operatorname{STD}=5124.369$

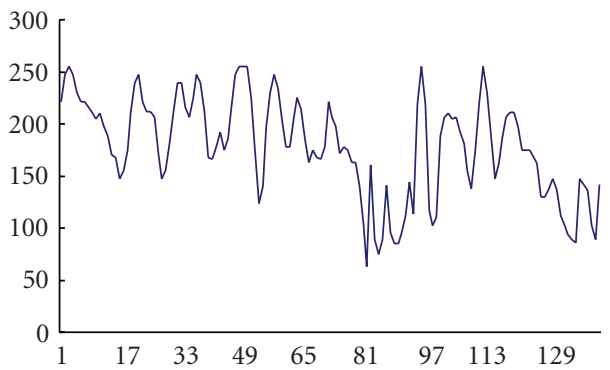

(c) Enhanced Lee Filter, mean $=133.078$, STD $=$ 3138.857

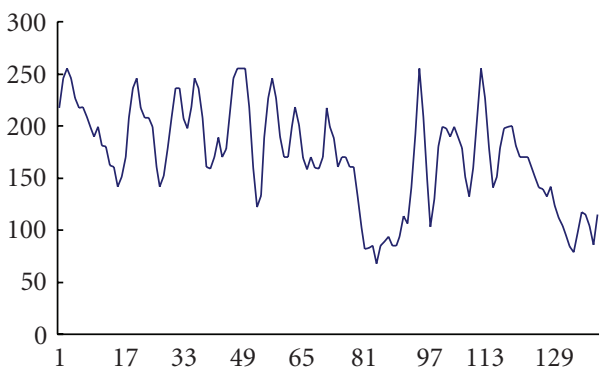

(e) Forest Filter, mean $=127.445$, STD $=$ 2892.149

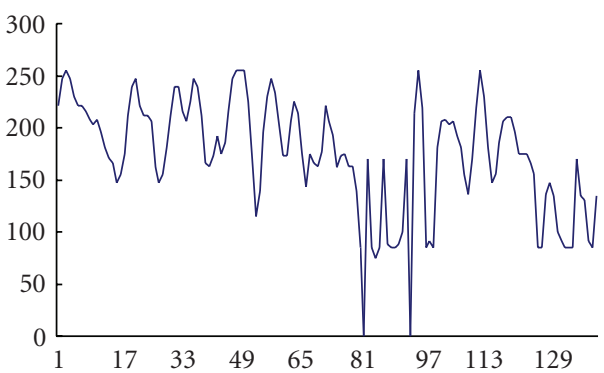

(g) Gamma Filter, mean $=128.3086$, STD $=$ 3662.987

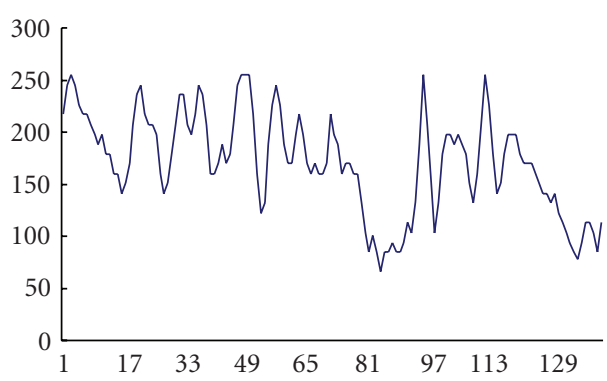

(b) Lee Filter, mean $=126.722, \operatorname{STD}=2967.306$

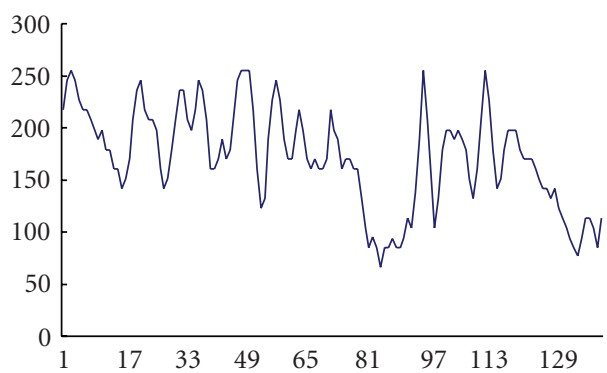

(d) Kuan Filter, mean $=127.230$, STD $=$ 2926.076

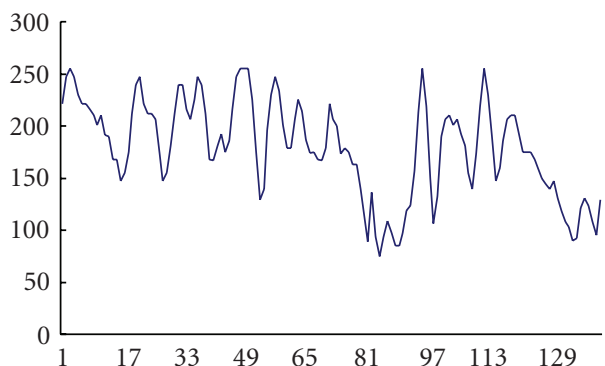

(f) Enhanced Forest Filter, mean $=134.298$, STD $=$ 2943.917

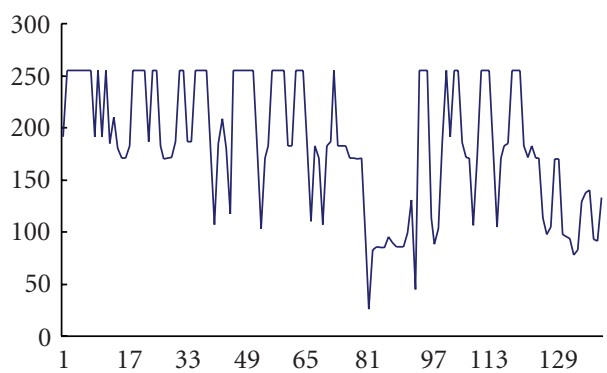

(h) Level set filter, mean $=131.3789$, $\mathrm{STD}=$ 4891.829

FIgURE 5: The profiles of Region D resulted from the proposed level set-based filter and conventional approaches.

The evolution speed is switched between $\min (\kappa, 0)$ and $\max (\kappa, 0)$, and diffusion is turned on or off depending the scale of the noise that wants to remove. $\kappa$ is simplified as

$$
\kappa= \begin{cases}\max (\kappa, 0) & \text { if }\left(\bar{I}_{s}<I_{\text {threshold }}\right), \\ \min (\kappa, 0) & \text { if }\left(\bar{I}_{s}>I_{\text {threshold }}\right),\end{cases}
$$

where $I_{\text {threshold }}$ is calculated as the average of pixel intensities along the direction perpendicular to the gradient at the extrema of the local neighborhood within the filter window $\eta_{s}$. Comparison between the neighborhood average and the threshold is used to select the right speed function to use. Flow under $\min (\kappa, 0)$ would preserve some of the structure of the curve, while flow under $\max (\kappa, 0)$ completely diffuses away all of the information. This switch prevents the unwanted diffusion of the proposed curvature flow approach. In other words, a speed of $\kappa_{\max }$ will cause small dark regions in a predominantly light region to shrink. Conversely, a speed of $\kappa_{\min }$ will cause light regions in a predominantly dark region to shrink. 
TABLE 1: Mean, standard deviation and error standard deviation for regions A, B, and C.

\begin{tabular}{|c|c|c|c|}
\hline & A Region & B Region & Vertical line of $x=417$ \\
\hline & Raw data & Raw data & Raw data \\
\hline \multirow{3}{*}{ Raw image } & Mean: 186.515 & Mean: 102.284 & Mean: 115.445 \\
\hline & Std: 7356.633 & Std: 557.088 & Std: 3807.465 \\
\hline & Error std: 0.000 & Error std: 0.000 & Error std: 0.000 \\
\hline \multirow{3}{*}{ Degraded image by speckle } & Mean: 168.146 & Mean: 96.008 & Mean: 107.806 \\
\hline & Std: 6474.725 & Std: 1063.434 & Std: 3551.714 \\
\hline & Error std: 1694.889 & Error std: 576.947 & Error std: 864.951 \\
\hline \multirow{3}{*}{ Lee Filter } & Mean: 167.054 & Mean: 95.558 & Mean: 106.755 \\
\hline & Std: 5318.111 & Std: 508.609 & Std: 2891.598 \\
\hline & Error std: 796.122 & Error std: 110.071 & Error std: 285.072 \\
\hline \multirow{3}{*}{ Enhanced Lee Filter } & Mean: 170.137 & Mean: 98.565 & Mean: 109.898 \\
\hline & Std: 5490.712 & Std: 521.443 & Std: 2975.532 \\
\hline & Error std: 725.142 & Error std: 85.426 & Error std: 283.281 \\
\hline \multirow{3}{*}{ Kuan Filter } & Mean: 167.667 & Mean: 96.006 & Mean: 107.250 \\
\hline & Std: 5258.601 & Std: 508.466 & Std: 2866.156 \\
\hline & Error std: 756.300 & Error std: 104.209 & Error std: 266.664 \\
\hline \multirow{3}{*}{ Frost Filter } & Mean: 167.875 & Mean: 95.998 & Mean: 107.378 \\
\hline & Std: 5205.227 & Std: 509.813 & Std: 2849.575 \\
\hline & Error std: 737.993 & Error std: 104.189 & Error std: 253.703 \\
\hline \multirow{3}{*}{ Enhanced Frost Filter } & Mean: 170.349 & Mean: 98.752 & Mean: 110.125 \\
\hline & Std: 5432.889 & Std: 510.254 & Std: 2965.519 \\
\hline & Error std: 705.102 & Error std: 76.273 & Error std: 268.414 \\
\hline \multirow{3}{*}{ Gamma Filter } & Mean: 169.335 & Mean: 97.692 & Mean: 108.734 \\
\hline & Std: 5617.564 & Std: 555.922 & Std: 3026.878 \\
\hline & Error std: 777.002 & Error std: 113.264 & Error std: 326.011 \\
\hline \multirow{3}{*}{ Level set Filter } & Mean: 181.439 & Mean: 90.712 & Mean: 105.244 \\
\hline & Std: 7673.753 & Std: 841.549 & Std: 4245.700 \\
\hline & Error std: 420.243 & Error std: 323.605 & Error std: 376.056 \\
\hline
\end{tabular}

3.4. Filter Framework. The framework for proposed level set filter is illustrated in Figure 1. Unlike the common filters based on statistical average in a fixed window, this algorithm mainly relies on the theory of propagating interface, that is, Level set, controlled by partial differential equations. Thus, the smoothed image is obtained only by an iterative process not a convolution or nonstationary, linear filter, and more iterations will result in further smoothing. The main process can be summarized as follows.

(1) Firstly, this filter requires four parameters used in the computation of the level set evolution, the number of iterations to be performed, the time step and the vicinity range, and the scale of the noise that one wants to remove.

(2) Secondly, compute curvature-driven model and adaptive filter coefficient, respectively.
(3) Thirdly, for preserving finer detailed structures in images, switch curvature flow according to min-max flow.

(4) Continuously update pixel intensity by function (18).

(5) This approach adjusts the value of each pixel based on the iteration.

\section{Experimental Results}

In this section, we firstly test the proposed level set filter using simulated data, then, demonstrate the algorithm on land and ocean SAR data. In each experiment, we compare the results of the proposed level set filter with those of six existing schemes, that is, Lee filter, Enhanced Lee Filter, Kuan Filter, Frost Filter, Enhanced Frost Filter, Gamma Filter. We examine the mean preservation error, the standard deviation and error standard deviation 
reduction to quantify the performance of algorithms in different regions.

Mean, standard deviation and error standard deviation are defined by

$$
\begin{aligned}
\bar{I}_{g} & =\sum_{\text {pixel }(i, j) \in g}^{n} \frac{\hat{I}_{(i, j)}}{n}, \\
\operatorname{STD} & =\sum_{\text {pixel }(i, j) \in g}^{n} \frac{\left(\hat{I}_{(i, j)}-\bar{I}_{g}\right)^{2}}{n}, \\
\text { Error STD } & =\sum_{\text {pixel }(i, j) \in g}^{n} \frac{\left(\hat{I}_{(i, j)}-I_{(i, j)}\right)^{2}}{n},
\end{aligned}
$$

where $\hat{I}$ is a result pixel after filter and $I$ is a supposed true pixel. Usually, STD represents information complexity while error standard deviation shows difference between result image after filter the degraded image and theoretically true image, that is, the initial image.

Furthermore, the profile graph was usually used to illustrate performance of noise smoothing and edge preservation [14]. In this paper, profiles were select by arbitrary line in the image, where $x$-axis represents line distance and $y$-axis is the intensity of pixels.

It should be noted that although the evolution at the boundary is slow, some diffusion still may occur. In addition, considering more iterations will result in further smoothing and will linearly increase time consumption [18], thus the small number of iterations is selected to stop the computation of the level set evolution. In the following experiments, the filter was run with less than 5 iterations, $5 \times$ 5 pixels vicinity range for intensity model.

4.1. Simulation Results. In comparison with the above traditional SAR filters and proposed method in this paper, simulation image (Figure 2(b)), which comes from shore scene (Figure 2(a)), is used to test the effectiveness in Figure 2.

Firstly, the initial image is degraded by multiplicative and random noise in Figure 2(b). Then based on degraded image, result images computed by traditional methods such as Lee filter, Enhanced Lee Filter, Kuan Filter, Frost Filter, Enhanced Frost Filter, Gamma Filter and proposed level set filter are denoted in Figures 2(c), 2(d), 2(e), 2(f), 2(g), 2(h), and 2(i), respectively.

Secondly, initial undegraded image is supposed as theoretically true value, so mean, standard deviation, and error standard deviation of result images in different areas such as region A with image boundary and annotation, region $B$ with shore and village and vertical line $\mathrm{C}$ (where $x$ axis equal to 417) are summarized in Table 1.

Comparing the traditional filter results of region $\mathrm{A}$ in Figure 2, it is clearly that noise in the margin is removed perfectly by proposed level set filter and the edges of image and annotation are preserved effectively (Figure $2(\mathrm{i})$ versus Figures 2(c), 2(d), 2(e), 2(f), 2(g), 2(h)).
At the same time, the profiles of pixels intensity along vertical line C (where $y$ axis is between 347 and 495) in result images are plotted in Figure 3. For all It illustrates that the intensity shape of the proposed level set filter is distinguished from traditional filter results, and keeps the boundaries with smoothing noise inner and outer region perfectly, and more close to true value than others (Figure 3(i) versus Figures 3(c), 3(d), 3(e), 3(f), 3(g), 3(h)).

In addition, because the level set filter not only preserves edges but also enhances edges by inhibiting diffusion across edges and allowing diffusion on either side of edge. So, standard deviation will become large in Table 1. Meanwhile, it appears that some edges are enhanced, that is, the difference of intensity value on either side of edge becomes large (Figure 3(i)). At a word, black will become more black while white more white on either side of edge.

4.2. ERS-2 SAR Image Results. To test the effectiveness of our level set filter in different environments, the following two SAR images were computed by the above traditional filters and proposed level set filter, respectively.

(i) ERS-2 SAR (Precision Image product with number of looks 3) PRI image with $559 \times 649$ pixels on the Shangdong peninsula landscape in Figure 4. In addition, profile of partial pixels intensity along vertical line D is plotted in Figure 5.

(ii) ERS-2 SAR (Precision Image product with number of looks 3) PRI image with $295 \times 360$ pixels at the South China Sea seascape in Figure 6. In addition, profile of partial pixels intensity along vertical line $\mathrm{E}$ is plotted in Figure 7.

In Figure 4, vertical line D is cross some croplands and forms these show comb structure in Figure 5. Figures 4(b), 4(c), 4(d), 4(e), 4(f), and 4(g) demonstrate that the above traditional filters cannot preserve the narrow strips perfectly due to only inhibiting smoothing near edges and not supporting direction filter. In contrast, proposed level set filter (Figure 5(h)) keeps up the large strips and smoothing the small strips.

In Figure 6, besides high noise coming from the inherent nature of radar backscatter, due to the dampening effects of Bragg wave on the sea generated by low backscatter cross section of the surface, SAR images usually have a high noise. Figure 6 shows that the result of proposed filter is near to the result of traditional methods such as Lee filter, Enhanced Lee Filter, Kuan Filter, Frost Filter, Enhanced Frost Filter and Gamma Filte (Figure 6(h) versus Figures 6(b), 6(c), 6(d), 6(e), 6(f), and 6(g)).

\section{Conclusions}

In this paper, a filter, which is based on level set, has been explored for speckle removal in SAR images. As we know, Level set is an efficient numerical technique 


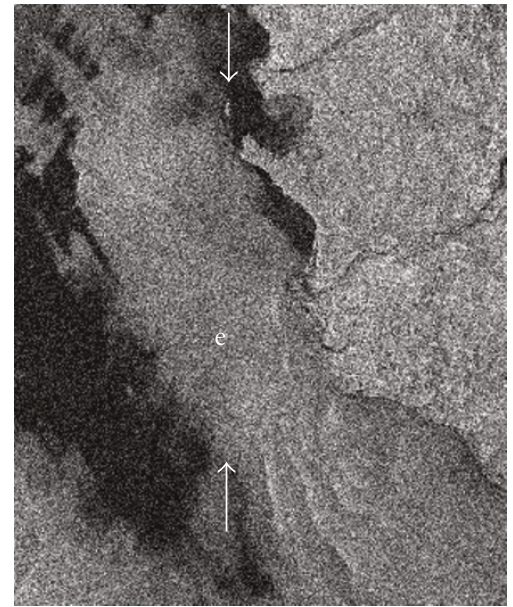

(a) Raw data

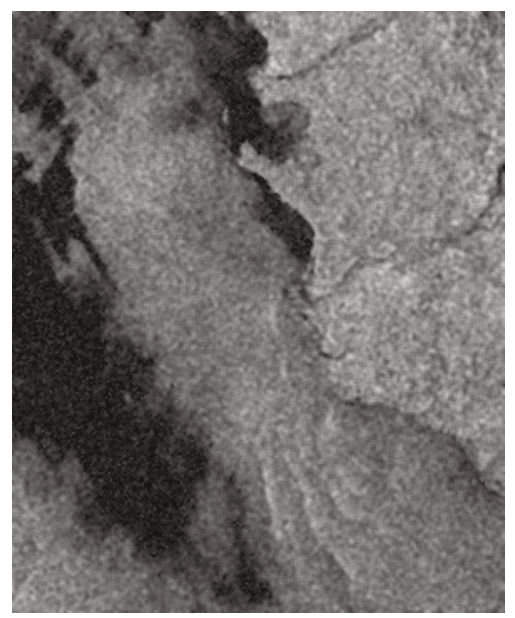

(d) Kuan Filter

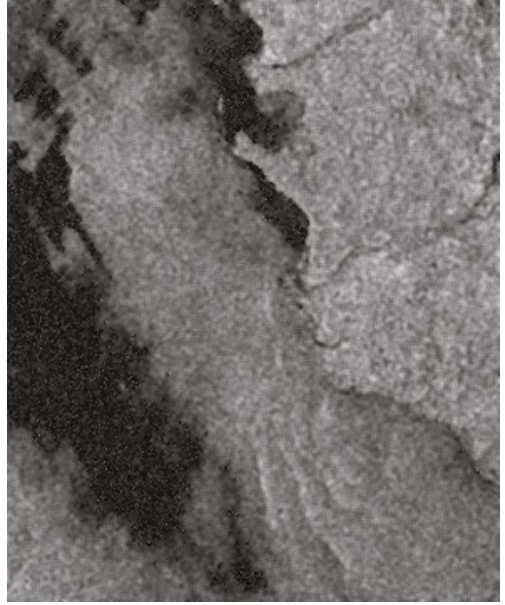

(b) Lee Filter

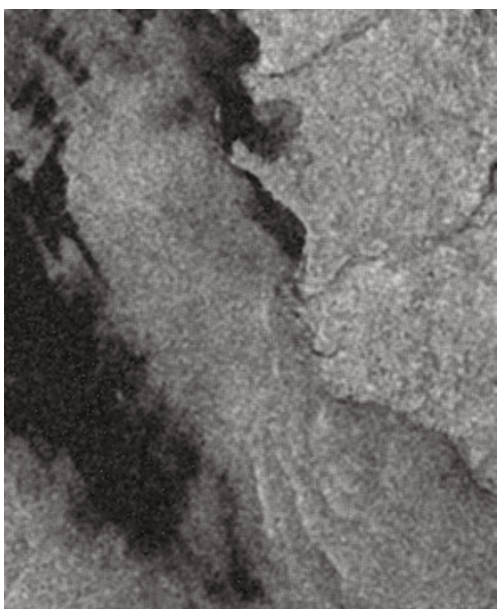

(e) Frost Filter

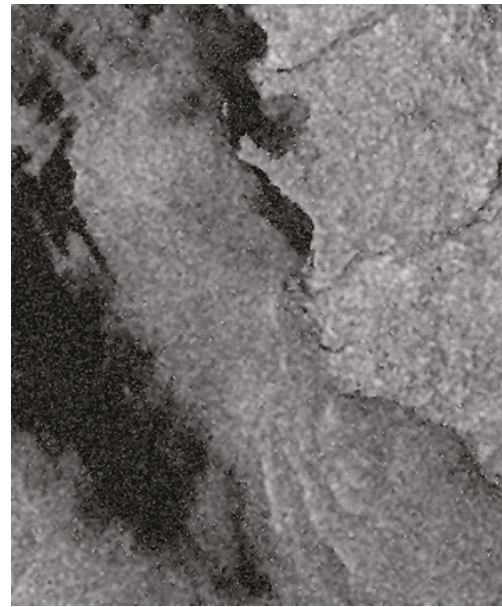

(c) Enhanced Lee Filter

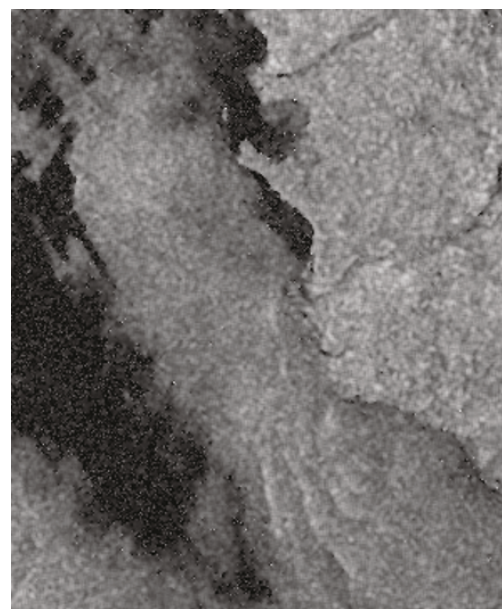

(f) Enhanced Frost Filter

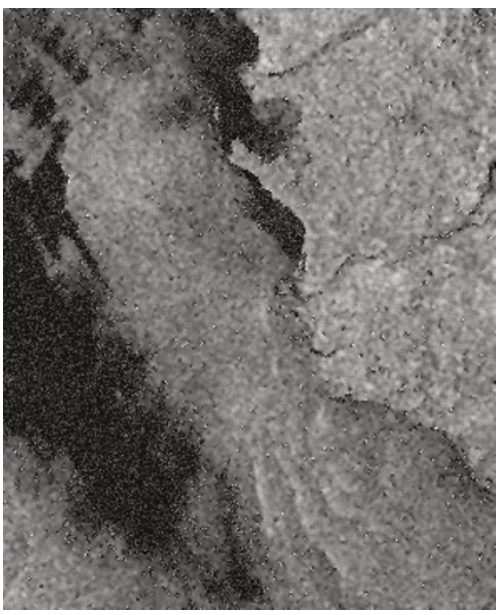

(g) Gamma Filter

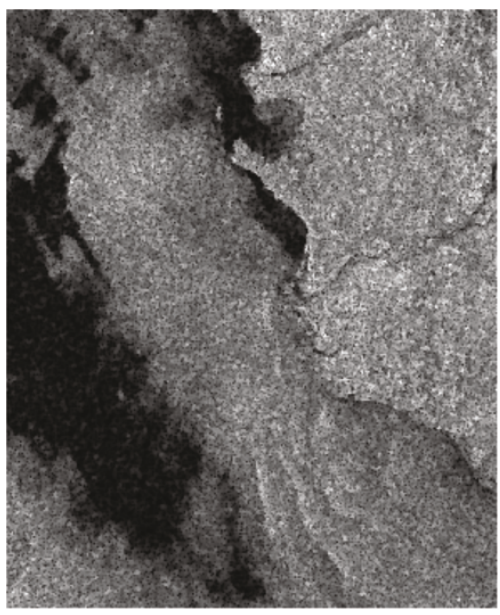

(h) Level set filter

FIGURE 6: The proposed level set-based filter in contrast to the conventional approaches of seascape ERS-2 SAR image.

while Lee filter is a classical speckle noise filter. What is new here is the correspondence between these two types of frameworks, moreover, for speckle filtering purposes. Unlike other existing speckle reducing filters, it is based on the theory of flow propagation and Lee filter, which uses intensity and curvature as the front advancing impetus to modify noise by differential equation iterations. According to theoretical analysis, imagery experiment including 


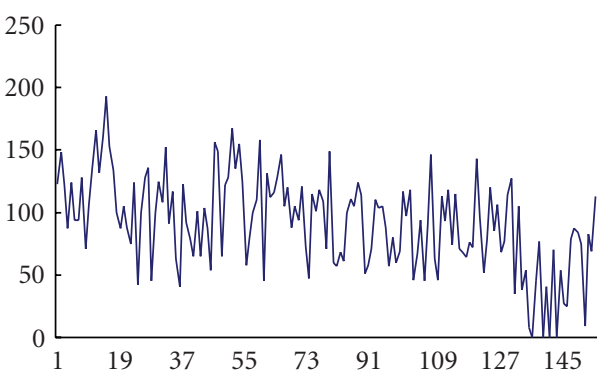

(a) Raw data, mean $=50.181, \operatorname{STD}=1978.941$

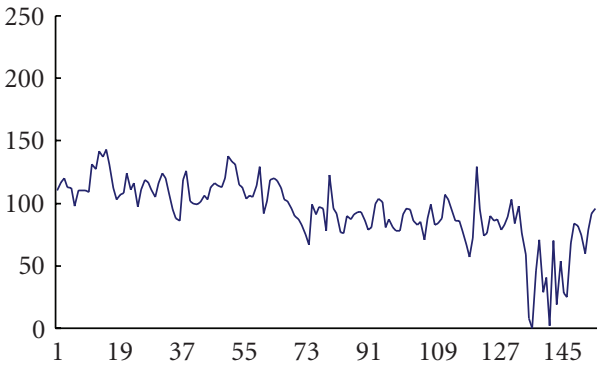

(c) Enhanced Lee Filter, mean $=51.406$, STD $=$ 1640.053

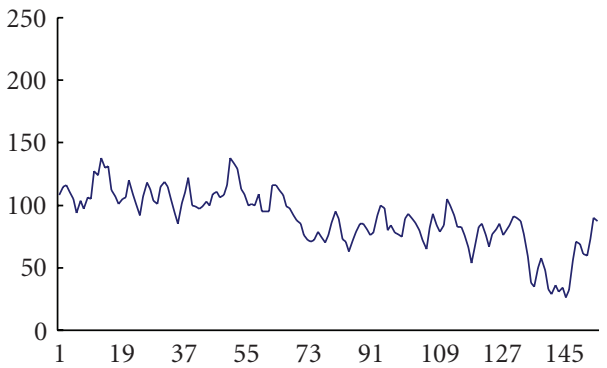

(e) Frost Filter, mean $=48.990$, STD $=1458.836$

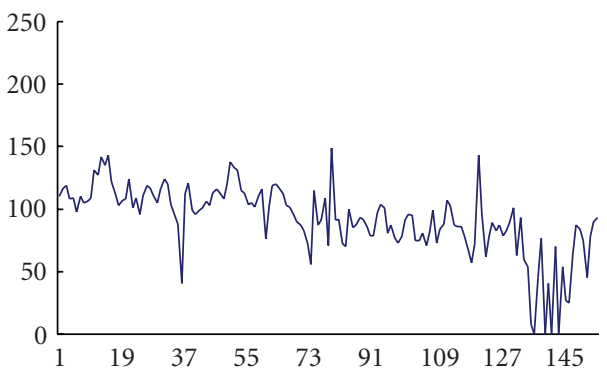

(g) Gamma Filter, mean $=50.437$, STD $=$ 1657.338

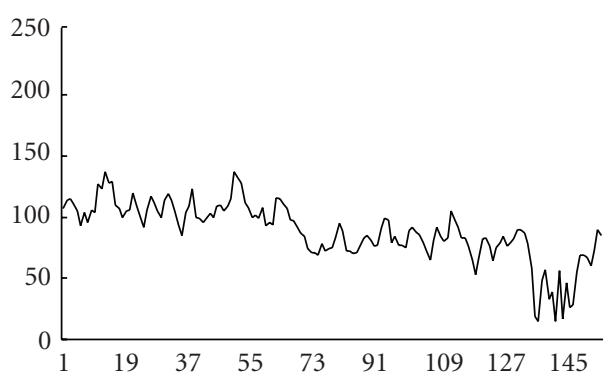

(b) Lee Filter, mean $=48.662, \mathrm{STD}=1456.012$

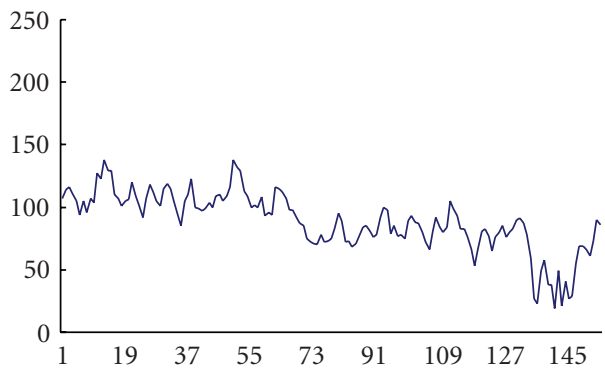

(d) Kuan Filter, mean $=48.882$, STD $=1461.612$

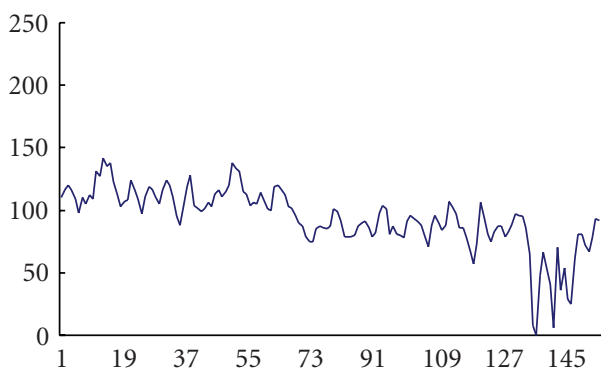

(f) Enhanced Frost Filter, mean $=51.333$, STD $=$ 1598.938

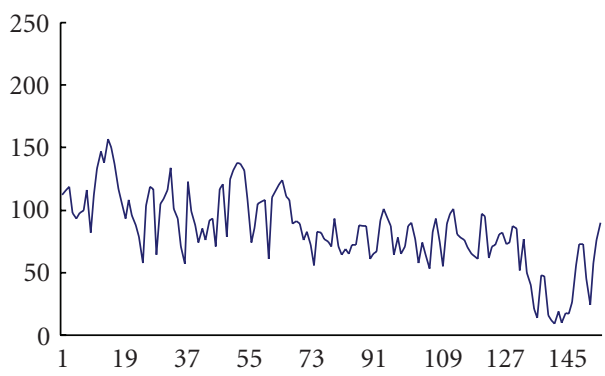

(h) Level set filter, mean $=47.197$, $\operatorname{STD}=$ 1606.520

FIGURE 7: The profiles of Region E resulted from the proposed level set-based filter and conventional approaches.

simulation and landscape, and seascape of ERS-2 SAR images, experimental results reveal that the proposed algorithm provides superior performance in comparison to the conventional speckle filters, such as Lee filter, Enhanced Lee Filter, Kuan Filter, Frost Filter, Enhanced Frost Filter and Gamma Filter, in terms of both smoothing speckle inner or outer uniform regions and preserving (sometimes enhancing) edges and detail features. We hope that the method described in this paper provides a basis for further exploration of level set in remotely sensed image processing.

\section{Acknowledgment}

This research was funded by Ministry of Science and Technology of PRC under Contract no. 2006AA12Z116, and their support is gratefully acknowledged. 


\section{References}

[1] J.-S. Lee, "Speckle suppression and analysis for synthetic aperture radar images," Optical Engineering, vol. 25, no. 5, pp. 636-643, 1986.

[2] A. Lopes, E. Nezry, R. Touzi, and H. Laur, "Maximum a posteriori filtering and first order texture models in SAR images," in Proceedings of the International Geoscience and Remote Sensing Symposium (IGARSS '90), pp. 2409-2412, College Park, Md, USA, 1990.

[3] D. T. Kuan, A. A. Sawchuk, T. C. Strand, and P. Chavel, "Adaptive restoration of images with speckle," IEEE Transactions on Acoustics, Speech, and Signal Processing, vol. 35, no. 3, pp. 373383, 1987.

[4] V. S. Frost, J. Stiles, K. Shanmugan, and J. Holtzmann, "A model for radar images and its application to adaptive digital filtering of multiplicative noise," IEEE Transactions on Pattern Analysis and Machine Intelligence, vol. 4, pp. 157-166, 1982.

[5] P. Perona and J. Malik, "Scale-space and edge detection using anisotropic diffusion," IEEE Transactions on Pattern Analysis and Machine Intelligence, vol. 12, no. 7, pp. 629-639, 1990.

[6] L. Alvarez and L. Mazorra, "Signal and image restoration using shock filters and anisotropic diffusion," SIAM Journal on Numerical Analysis, vol. 31, no. 2, pp. 590-605, 1994.

[7] Y. Yu and S. T. Acton, "Speckle reducing anisotropic diffusion," IEEE Transactions on Image Processing, vol. 11, no. 11, pp. 1260-1270, 2002.

[8] S. Aja-Fernandez and C. Alberola-Lopez, "On the estimation of the coefficient of variation for anisotropic diffusion speckle filtering," IEEE Transactions on Image Processing, vol. 15, no. 9, pp. 2694-2701, 2006.

[9] A. Achim, P. Tsakalides, and A. Bezerianos, "SAR image denoising via Bayesian wavelet shrinkage based on heavytailed modeling," IEEE Transactions on Geoscience and Remote Sensing, vol. 41, no. 8, pp. 1773-1784, 2003.

[10] J. R. Sveinsson and J. A. Benediktsson, "Almost translation invariant wavelet transformations for speckle reduction of SAR images," IEEE Transactions on Geoscience and Remote Sensing, vol. 41, no. 10, pp. 2404-2408, 2003.

[11] T. F. N. Kanaa, E. Tonye, G. Mercier, et al., "Detection of oil slick signatures in SAR images by fusion of hysteresis thresholding responses," in Proceedings of the International Geoscience and Remote Sensing Symposium (IGARSS '03), pp. 2750-2752, Toulouse, France, July 2003.

[12] Y. Wu and H. Maître, "Smoothing speckled synthetic aperture radar images by using maximum homogeneous region filters," Optical Engineering, vol. 31, no. 8, pp. 1785-1792, 1992.

[13] R. Touzi, "A review of speckle filtering in the context of estimation theory," IEEE Transactions on Geoscience and Remote Sensing, vol. 40, no. 11, pp. 2392-2404, 2002.

[14] J.-S. Lee, R. G. Mitchell, L. S. Dale, E. Pottier, and L. FerroFamil, "Scattering-model-based speckle filtering of polarimetric SAR data," IEEE Transactions on Geoscience and Remote Sensing, vol. 44, no. 1, pp. 176-187, 2006.

[15] E. Trouvé, Y. Chambenoit, N. Classeau, and P. Bolon, "Statistical and operational performance assessment of multitemporal SAR image filtering," IEEE Transactions on Geoscience and Remote Sensing, vol. 41, no. 11, pp. 2519-2530, 2003.

[16] S. Osher and J. A. Sethian, "Fronts propagating with curvature-dependent speed: algorithms based on HamiltonJacobi formulations," Journal of Computational Physics, vol. 79, no. 1, pp. 12-49, 1988.
[17] B. Huang, H. Li, and X. Huang, "A level set method for oil slick segmentation in SAR images," International Journal of Remote Sensing, vol. 26, no. 6, pp. 1145-1156, 2005.

[18] X. Huang, B. Huang, and H. Li, "A fast level set method for synthetic aperture radar ocean image segmentation," Sensors, vol. 9, no. 2, pp. 814-829, 2009.

[19] J. A. Sethian, Level Set Methods and Fast Marching Methods: Evolving Interfaces in Computational Geometry, Fluid Mechanics, Computer Vision and Materials Science, Cambridge University Press, Cambridge, UK, 1999.

[20] L. Rudin, S. Osher, and E. Fatemi, "Nonlinear total variation based noise removal algorithm," in Modelisation Matematiques pour le Traitement D'images, pp. 149-179, INRIA, 1992.

[21] G. Sapiro and A. Tannenbaum, "Image smoothing based on affine invariant flow," in Proceedings of the Conference on Information Sciences and Systems, Johns Hopkins University, 1993.

[22] R. T. Whitaker and X. Xue, "Variable-conductance, level-set curvature for image denoising," in Proceedings of the IEEE International Conference on Image Processing, pp. 142-145, IEEE, Thessalonik, Greece, October 2001. 